\title{
Intelligent recognition of colorectal cancer combining application of computer-assisted diagnosis with deep learning approaches
}

\author{
Akella S. Narasimha Raju1, Kayalvizhi Jayavel ${ }^{1}$, Tulasi Rajalakshmi ${ }^{2}$ \\ ${ }^{1}$ Department of Networking and Communications, School of Computing, SRM Institute of Science and Technology, Chennai, India \\ ${ }^{2}$ Department of Electronics and Communications, School of Electrical and Electronics Engineering, SRM Institute of Science and \\ Technology, Chennai, India
}

\begin{tabular}{l} 
Article Info \\
\hline Article history: \\
Received Feb 22, 2021 \\
Revised Jun 19, 2021 \\
Accepted Jun 29, 2021 \\
\hline
\end{tabular}

Keywords:

Colorectal cancer

Computer assisted diagnosis

Convolutional neural network

Deep learning

Tumor

\begin{abstract}
The malignancy of the colorectal testing methods has been exposed triumph to decrease the occurrence and death rate; this cancer is the relatively sluggish rising and has an extremely peculiar to develop the premalignant lesions. Now, many patients are not going to colorectal cancer screening, and people who do, are able to diagnose existing tests and screening methods. The most important concept of this motivation for this research idea is to evaluate the recognized data from the immediately available colorectal cancer screening methods. The data provided to laboratory technologists is important in the formulation of appropriate recommendations that will reduce colorectal cancer. With all standard colon cancer tests can be recognized agitatedly, the treatment of colorectal cancer is more efficient. The intelligent computer assisted diagnosis (CAD) is the most powerful technique for recognition of colorectal cancer in recent advances. It is a lot to reduce the level of interference nature has contributed considerably to the advancement of the quality of cancer treatment. To enhance diagnostic accuracy intelligent $\mathrm{CAD}$ has a research always active, ongoing with the deep learning and machine learning approaches with the associated convolutional neural network $(\mathrm{CNN})$ scheme.
\end{abstract}

This is an open access article under the CC BY-SA license.

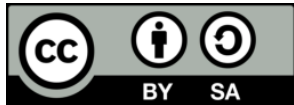

\section{Corresponding Author:}

Akella S. Narasimha Raju

Department of Networking and Communications, School of Computing, SRM Institute of Science and

Technology

Kattankalattur, Chennai, Tamilnadu, 603203, India

Email: ar9488@srmist.edu.in

\section{INTRODUCTION}

Colorectal cancer (CRC) is the most ubiquitous type of gastrointestinal (GI) malignancy. Different people in our world are going to be struggling with this cancer. This kind of cancer has taken a third place where people can be a cause of malignancy around the world. At the spot of death rate in adult males, this colorectal cancer is the third leading source around the globe, in this case, first and second places of risk dominating by prostate cancer and lung cancers. At the stage of death rate in women, this colorectal cancer is the second leading source around the globe, the first death risk dominating by the breast cancer.

Comparing with the world population, India is the second highest population among the world, but India has a low incidence rate of CRC, it has been calculated since last five years reports it has been 87 per 100,000 populations. Due to culture and differences in eating patterns, lifestyles are an indicator of being in control of the low incidence of CRC among developing countries. All over the world, the most important 
things to happen about CRC's incidence with obesity, which could be a risk issue for CRC. Among the elderly, youth also get evidence of increased CRC incidence. In India, $7.45 \%$ of youth was impacted by CRC. Among the global population, about $21 \%$ of the population could be affected by this CRC. Over the last 50 years, CRC has been targeting people around the world. Nowadays it is producing plenty of problems for people who are holding out in the western states are recognized like their lifestyle factors like obesity, smoking, drinking and dietary aspects. In the 2020, there will be approximately 18,000 (12\%) of cases relate to colorectal cancer diagnosed in our population, according to Global Burden of Cancer (GLOBOCAN) statistics [1], in 2020 around the world 1931590 cases and in India 65,358 cases new colorectal cancer. In India, $4.9 \%$ of observed cases occurred [2]. The estimated range of recent colorectal cancer cases worldwide and in India as shown in Figure 1 and Figure 2.

\section{India}

\section{Source: Globocan 2020}

\section{Number of new cases in 2020, both sexes, all ages}

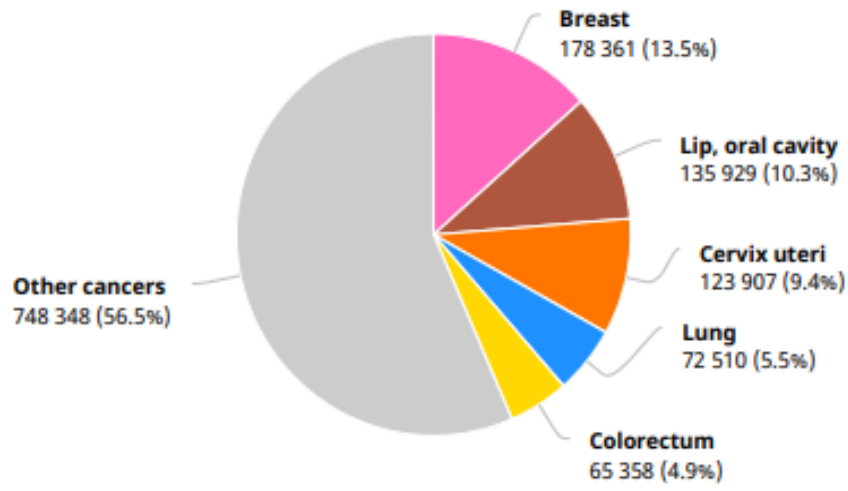

Total: 1324413

Figure 1. Estimation of the number of new cases globally in 2020

Estimated number of new cases in 2020, worldwide, both sexes, all ages

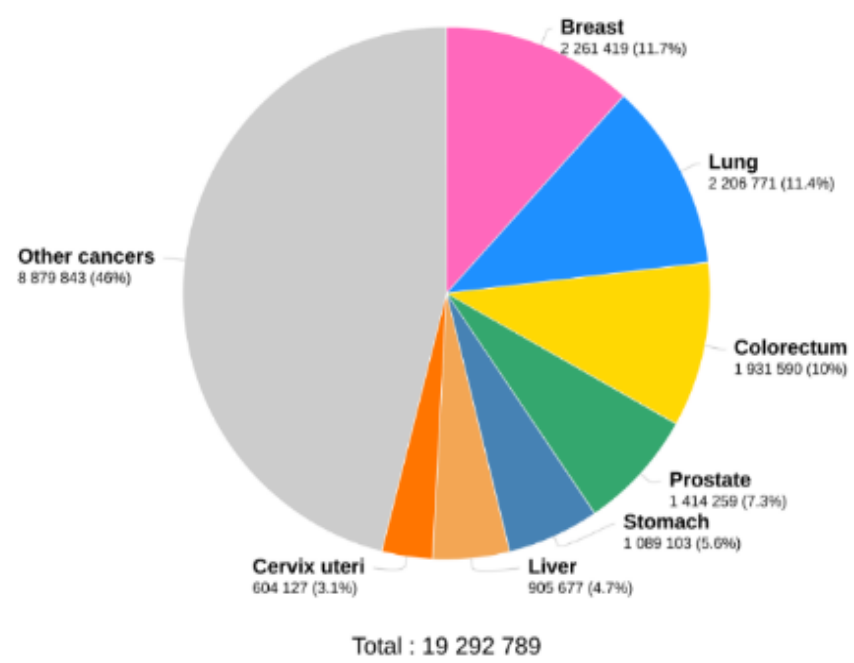

Figure 2. Estimated number of new occurrences in India in 2020 


\section{CURRENT PROCEDURES FOR THE IDENTIFICATION OF COLORECTAL CANCER}

\subsection{Large intestine and colon}

The large intestine is the major part of your digestive system. It sustains a physical process of the body food; the body does not use it, which becomes the waste. The colon prevails in the human intestine. It discharges into the part of the body, wherever a waste accumulates such as stool. The rectum is discharged into the anus, where the actions of the intestine leave the body. It is currently possible to get totally different means of screening for cancer of the large intestine in the world. The available screening methods are followed as:

\subsubsection{Sigmoidoscopy}

A flexible sigmoidoscopy uses a versatile luminous tube that is placed in the lower colon and rectum to visualize the tumor, the types of cancer abnormalities [3]. Throughout this practice, a physician will dispose of tumor tissue types for further examination. The testing process given permission to the eradication of tumors, and could be stopped colorectal cancer. However, if tumors are found and present in sigmoidoscopy is the best suggestion is colonoscopy throughout the colon?

\subsubsection{Colonoscopy}

A colonoscopy lets the doctor appear in the intestine and rectum despite the numbness of a patient. A soft, enlightened tube known as the "colonoscope" is placed in the rectal area and thus the entire colon to appear for the malignant tumor [4]. Throughout this procedure, a doctor is going to eradicate the tumors or different tissues for the testing process. Removal of tumors can also stop the colon and rectal malignancy.

\subsubsection{Wireless capsule endoscopy}

Wireless capsule endoscopy may even help with a tiny camera to capture images of a patient's internal colon [5]. A wireless capsule endoscopic camera is supported with a capsule the size of a vitamin to swallow. Because of the passage of the capsule into the channel, the camera captures a large number of images which are transmitted to a patient recorder lowering a belt around the waist.

\subsubsection{Computed tomography (CT) scan}

Computed tomography scans usually known as virtual endoscopy can be an exploratory test technique [4]. It requires explanation by a talented expert to produce the simplest outcomes. An expert might be a doctor focusing on getting and deciphering medical photos. Regular colonoscopy is a risk because of anaesthesia, if a person includes a block in the large bowel that ends a comprehensive examination.

\subsubsection{Magnetic resonance imaging (MRI) scans}

As with CT scans, magneitic resonant imaging (MRI) shows exhaustive images of sensitive tissues in the body [6]. But magnetic resonance imaging uses strong radio waves and magnets instead of X-rays. A contrast medium known as metal could also be injected into a vein before the scan to get clear photos. Magnetic resonance imaging is often not to investigate abnormal regions in the liver or brain and spinal cord which would be cancer are taking place.

\subsubsection{Fecal occult blood tests}

A fecal occult blood test found blood in the stool that could be a cancer of strong symptoms or tumors in the colon 4 . When a positive report is produced, a significant amount of blood is found in the stool. There will be causes other than bleeding from the colon in the stomach or higher gastrointestinal tract. We have two types of tests: guaiac (FOBT) and immunochemical (FIT). Tumors and malignancies do not bleed frequently, therefore FOBT should take numerous stool samples each year and may be recurrent each year. Yet this screening translates into a relatively small decline in colorectal cancer mortality.

\subsubsection{Double contrast barium enema (DCBE)}

Patients who have not taken the colonoscopy screening procedure may take the test with an enema containing barium [3]. Barium enema screening procedure facilitates the creation of the large intestinal area and rectal area differ on X-rays. It is followed by a series of radiographies of the colon and rectum. In general, physicians would recommend additional tests for barium enema outcomes. This is probably a smaller amount to see metastatic tumor polyps than endoscopy, flexible sigmoidoscopy, or CT colonoscopy.

\subsubsection{DNA testing of feces}

This stool DNA test analyses the deoxyribonucleic acid (DNA) of a stool sample from a patient to determine whether cancer exists [4]. It uses polymer modifications that take place in polyps and cancers to determine the need for an endoscopy. 


\section{DISCUSSION AMONGS THE IMAGING SCREENING MODALITIES}

Colorectal cancer is thought to be appropriate for screening because of the progression of carcinoma. Which screening method is the best and perfect that the test should also be accepted by most individuals? The latter 1990s, there were on two screening methods for detecting tumors for malignancies, they are colonoscopy and DCBE. New approaches such as CT scanning and MRI colonoscopy have recently been introduced. Table 1 presents the pros and cons of these four methodologies [7].

Table 1. Comparative analysis for colonoscopy DCBE, CT scan, and MRI

\begin{tabular}{ccccc}
\hline Intervention possible & Yes & No & No & No \\
\hline Colon ratio tested. & $95 \%$ & $95 \%$ & $100 \%$ & $100 \%$ \\
Cost & High & Low & High & High \\
Sedation required & Yes & No & No & No \\
Sensitivity to polyp detection $<1 \mathrm{~cm}$ & $75 \%$ & $70 \%$ & $40 \%$ & $60 \%$ \\
Sensitivity to polyp detection $>1 \mathrm{~cm}$ & $90 \%$ & $80 \%$ & $93 \%$ & $96 \%$ \\
Specificity to polyp and cancer detections & $100 \%$ & $95 \%$ & $97 \%$ & $99 \%$ \\
Risk & $1: 1000$ & $1: 25000$ & Undetermined & Undetermined \\
\hline
\end{tabular}

In this article, we discuss DCBE's sensitivity and specificity to colonoscopy. DCBE was $85 \%$ sensitive in detecting colon cancer. The sensitivity for the detection of tumors with a diameter of $1 \mathrm{~cm}$ or greater was $80 \%$ and the sensitivity for the detection of tumors less than $1 \mathrm{~cm}$ was 70 . DCBE has a specificity of $95 \%$ for polyps and cancer. Colonoscopy sensitivity for colon cancer detection was $95 \%$, sensitivity for tumors $1 \mathrm{~cm}$ or more was $90 \%$, and sensitivity for tumors less than $1 \mathrm{~cm}$ was $75 \%$. This study found that the sensitivity of colonoscopy is almost certainly no more than $10 \%$ higher than that of DCBE for cancer and 15\% higher than that of DCBE. An optimistic feature of colonoscopy is the possibility of performing an instant procedure, such as biopsy, in the same subroutine. Sedation plays a positive role in patient acceptance.

It is also important to achieve high specificity for computed tomography and colonoscopy by MRI. Therefore, with this article on likely patients, more than $75 \%$ would have a preference for traditional colonoscopy. According to the report, the majority of patients prefer colonoscopy, as shown in Figure 3.

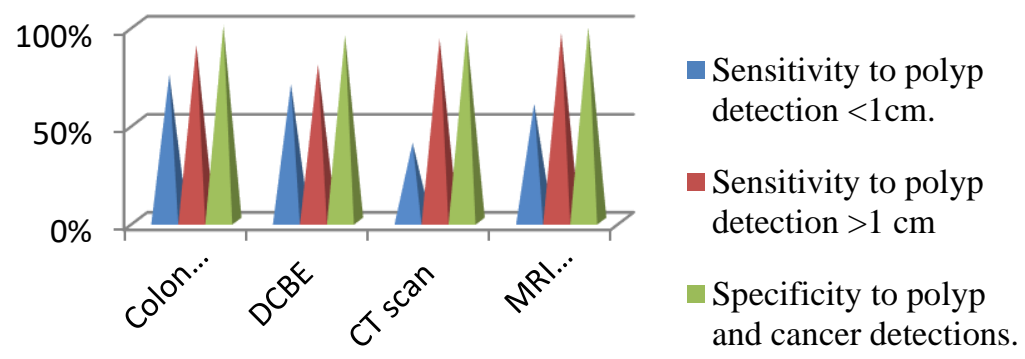

Figure 3. Statistical analysis of colonoscopy, DCBE, CT scan, and MRI

Finally, CT and MRI colonoscopy are new techniques in colon imaging that are comparable to conventional colonoscopy. However, these novel techniques for symptomatic patients only show results capable for tumor detection up to $1 \mathrm{~cm}$ in diameter. It is important to remember that in all research protocols, colonoscopy is strongly regarded as the characteristic criterion. This means that the other imaging modalities compared to colonoscopy will always be less preferable. The majority of patients were supportive of conventional colonoscopies. The intestinal cleansing diet is considered tedious, so from the point of view of patient acceptance, fecal marking techniques are promising. All these studies offer $100 \%$ accuracy from the conventional colonoscopy. This colonoscopy gives you lots of high-quality photos of discovering the disease.

\section{COMPUTER ASSISTED DIAGNOSIS (CAD)}

Prior to post-processing, input images should be evaluated for image quality and abnormalities using intelligent computer assisted diagnosis (CAD) [8]. In the detection of colorectal cancer, $\mathrm{CAD}$ is used. The block diagram for intelligent CAD [8] for detection of colorectal is shown in Figure 4. 
The colonoscopy technique delivers multiple image numbers in the form of image data sets [9]. Remember that these source image data sets should be of the highest quality possible. The larger the input images, the easier it is to detect the difference in the qualities of the pre-processing images. Source images should be carefully examined for filling defects and, if required, to improve the lesions. The concept of the intelligent CAD system is explained in the following steps.

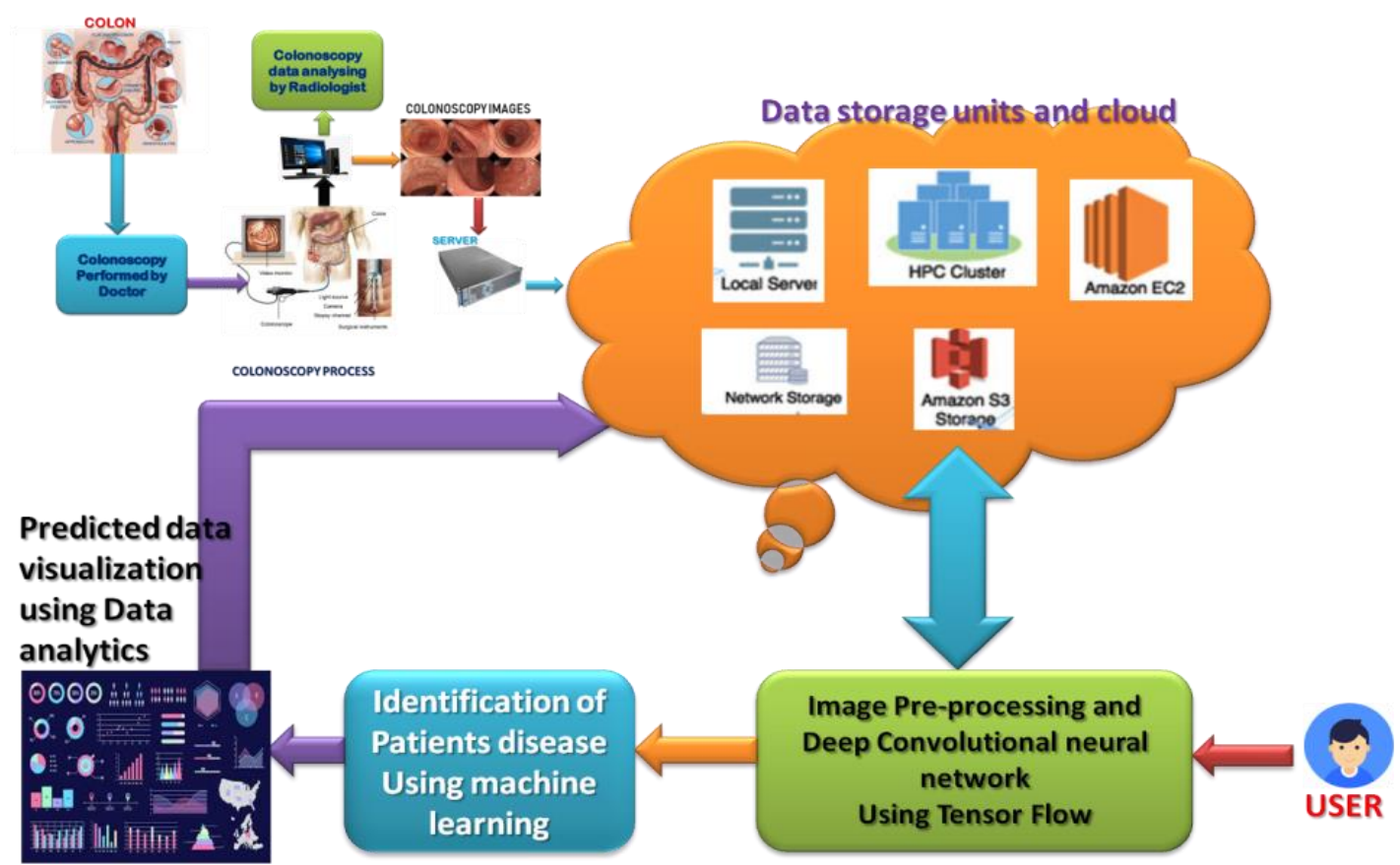

Figure 4. Block diagram for intelligent computer assisted diagnosis for detection of colorectal cancer

\subsection{Large intestine}

The large intestine has a length of approximately 1.5 meters, its main function is to absorb salts and water from the food consumed. It is not digestible food that can remain. The colon is the primary location of the large intestine; its real function is the absorption of salt and water. It can be divided into 4 parts: ascending colon, descending colon, sigmoid colon and rectal.

\subsection{Colonoscopy}

Colonoscopy is the screening method used to detect and identify tumors throughout the colon [10]. During this colonoscopy procedure, the doctor checks the abnormality for tumors. The physician checks throughout the colon with the camera fixed thin, light tube that is inserted in the starting point of the colon that is rectum. The camera generates the images that are monitored on the computer screen. After completion of the complete screening procedure, the computer gives the various sets of images captured by the camera from two full points. These images are available for detailed examination by the radiologist. The resulting images are then stored on the local server for upcoming references.

\subsection{Data storage and cloud storage}

Cloud storage [11], [12] is a critical component of this CAD. This enables you to analyse big data obtained from the date the image is stored from the local servers. Fast access to image data represents the data stored within the cloud. This is a very valuable and vital concept of cloud computing. As a result of a read and write operation, respectively, the amazon cloud system is the best choice for cloud storage.

The computing platform is the critical requirement for big data analytics. Hence, performing computational tasks, in parallel is to reduce the time with high performance calculations. Other GPUs and CPUs are introduced for parallel processing. Whereas Map reduce is primarily used by programmers for various calculation tasks for deep learning and machine learning techniques [13], [14]. Map reduce loads data back into memory from local servers and returns results from deep learning algorithms in imaging. The new methods implemented, could take advantage of the parallel processing capacities of a system. 


\subsection{Deep learning and machine learning steps}

\subsubsection{CNN trained to classify: architecture}

While performing the computer aided diagnosis (CAD) it's tend to fuse with the short designate the elements of a CNN. This is jointly which means it will be used to performing the CNN for classification [15], [16]. A CNN looks amazingly close to the usual neural networks among the feeling of being created by neurons with their different weights, biases, and activation functions. More precisely, the layout is shaped by a sequence of convolutions and aggregation layers ending in a highly connected neural network, as shown in Figure 5 .
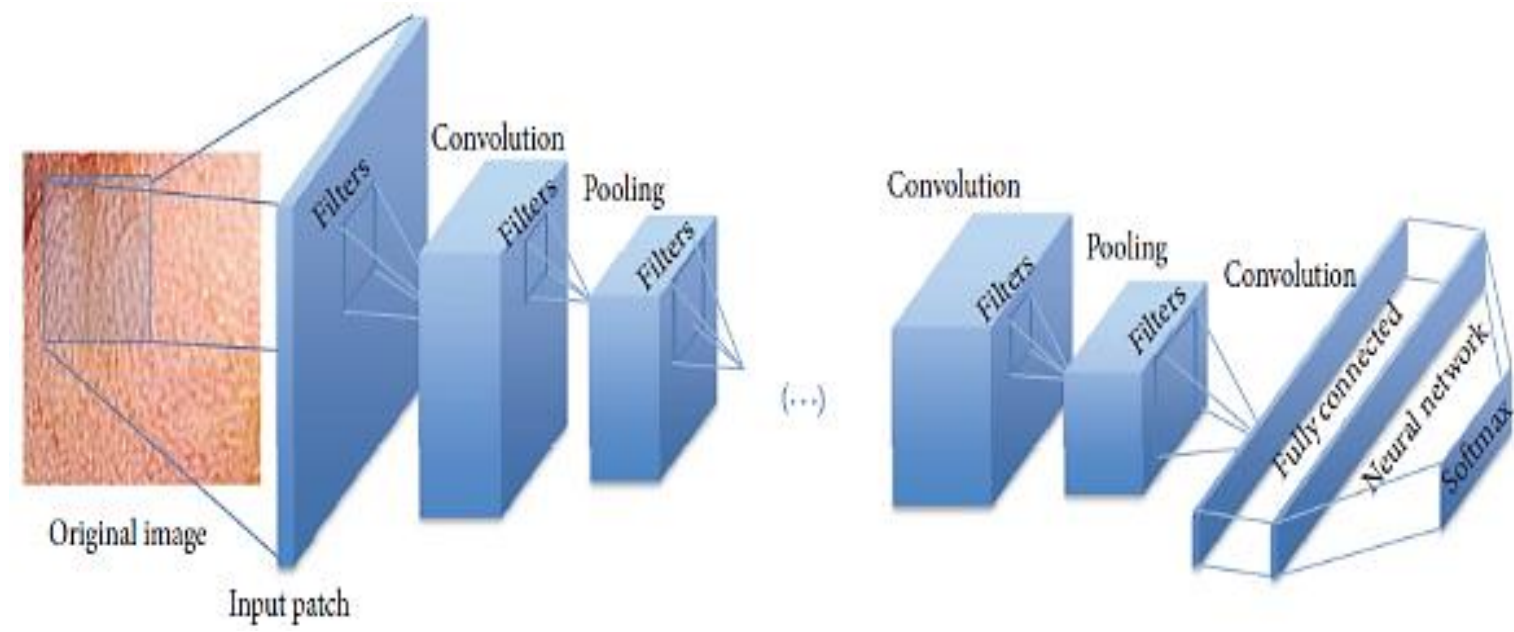

Figure 5. Image of the CNN architecture for the classification of colorectal tumors

The convolutional neural network (CNN) layout input is an image $m \times m \times d$ (or graph), that represents the input image dimension and $\mathrm{d}$ is that the diversity of channels that is also called as the depth of the image. The convolutional layer consists of $k$ learnable filters (also noted as Kernels) with size $n \times n \times d$ where $n \leq m$ that area unit convolved with the input image leading to the alleged activation maps or feature maps. Like traditional artificial neural networks (ANN), the outputs of the convolution layer are a unit subject to the associated activation operate, for example, the rectifier $(\operatorname{ReLU})$ operates on $\mathrm{f}(\mathrm{x})=\max (0, \mathrm{x})$, irrespective of the neuronal input. Later than convolution, a pooling layer is integrated to subsample the image using pooling functions of medium (medium) or maximum on $\mathrm{p} \times \mathrm{p}$ regions. These functions are won't to scale back the spatial attribute of the information among the subsequent layers upper layers and to offer a way of invariability for conversion so production overfitting management. In this convolution and pooling layers, the step needs to be specified; the larger the step, the lesser the overlap, thereby reducing the size of the output quantity. At the best of the $\mathrm{CNN}$ there is a fully connected layer as a daily multilayer neural network with the softmax feature that makes a grammatical likelihood distribution on the outputs. Following supervised training, the $\mathrm{CNN}$ is prepared to be used as a classifier or the feature extractor among learning transfer cases.

The model shown in Figure 6 colonoscopy image segmentation represents pre-processing, feature extraction and training of $\mathrm{CNN}$ and support vector machine (SVM), testing and generation of segmentation results. It's a three-stage process [17]-[19]: i) CNN and Integrated SVM are trained to the resulting tumor, ii) the labelled input and test image are entered to integrate the SVM classifier, iii) the iterative step connects CNN with the integrated SVM. A comprehensive classification process applied to the processed image: i) $\mathrm{CNN}$ has classified the pixels into a key zone, generating a sort of pre-segmentation which will be sent to the integrated SVM classifier. This will generate the pre-segmentation region of interest (ROI) [20]-[22], ii) the integrated SVM explores the neighbourhoods of the CNN output. Use CNN to re-classify a marked ROI. Repeat the steps to refine the segmentation findings [23]-[25].

\subsection{Visualization}

Segmented [26] results of the colonoscopy image will be analyzed with the objective of identifying colorectal cancer. Using the different visualization techniques, the information is then stored in public or private cloud storage. This could be accessed by different users like patients and healthcare professionals. 


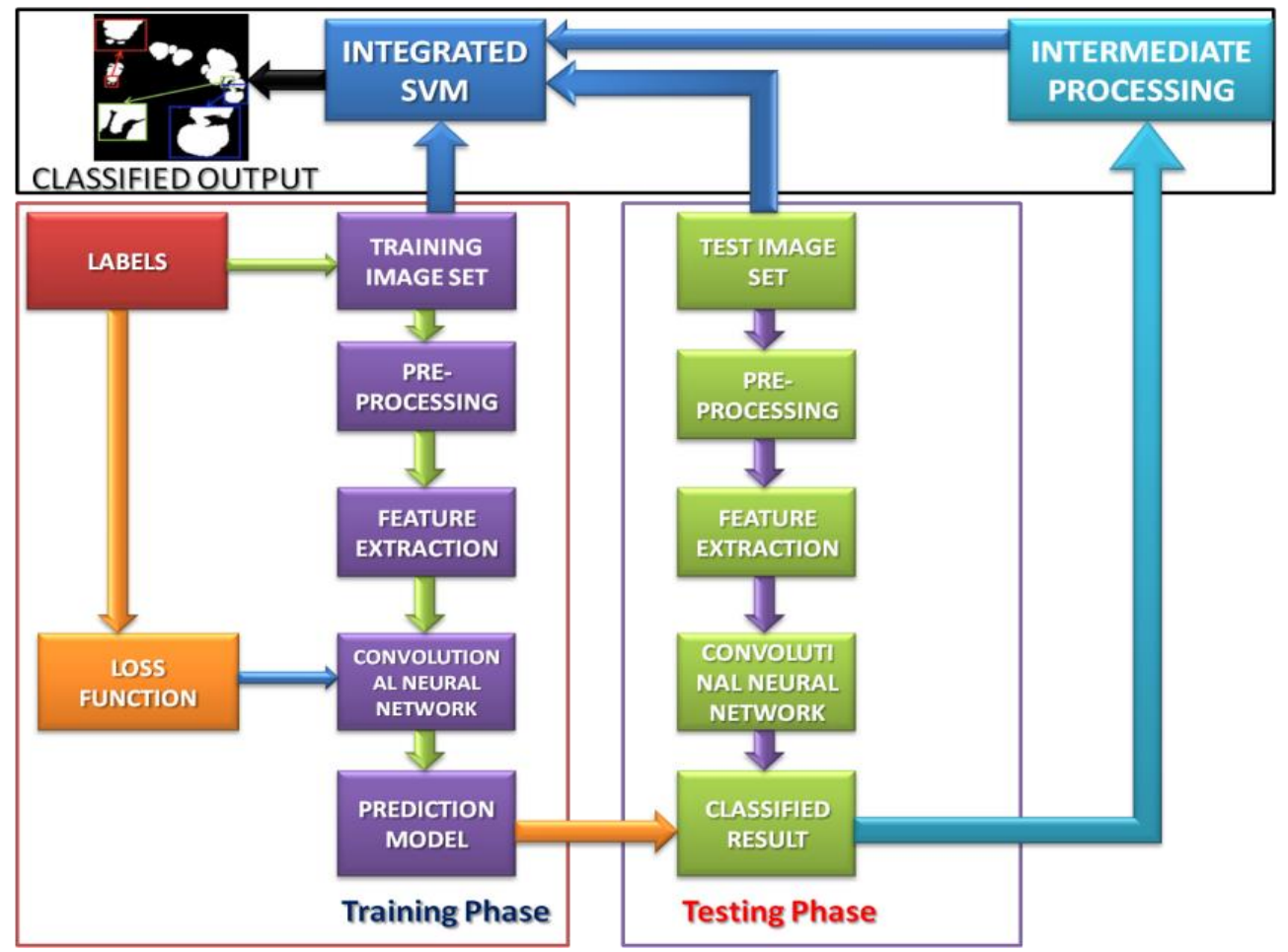

Figure 6. The deep learning and machine learning phases

\section{RESULTS AND DISCUSSION}

Cloud storage [11], [12] is a significant aspect of the CAD. This allows you to analyse the resulting big data from the date the image is stored from the local servers. Quick access to image data represents the data stored in the cloud. It is a very important and essential concept of cloud computing. Owing to a read and write operation, respectively, our existing personal google drive cloud system is the best choice for cloud storage for single set of data access. The computing platform is key to big data analysis. Thus, executing computational tasks, in parallel is to reduce the time with high performance calculations. Additional GPU and CPU are introduced for parallel processing.

\subsection{Kvasir 2 dataset}

In this experiment, the Kvasir 2 dataset was utilized, this dataset includes 8000 endoscopic gastrointestinal diseases and includes 8 different classes with 1000 images each. This data set consists of multiple sets of images in various categories including: i) anatomical references such as the z-line, pylorus and cerum, ii) pathologic results such as esophagitis, polyps and ulcerative cotis, iii) images with various resolutions between $720 \times 526$ and 1920x1072 pixels.

\subsection{Network training}

The set-up is based on Keras and the back-end is tensor flow. The training set serves to form the model and to learn the parameters. The validation set is used for optimizing and testing the model. During the machine training automatically adjust the learning rate and decide if early as a consequence to test the performance of a given training step.

We initialize the pre-trained weights of Resnet50 and use the stochastic gradient descent with a batch size of 201 and epochs of 50. The learning rate begins with 0.001 and is divided by 10 . Their core models the convolutional neural networks of deep learning and transfers learning to deep learning. It is possible to reach 93 percent accuracy shown in Figure 7.

A confusion matrix is a technique used to synthesize the efficiency of a classification algorithm. The accuracy of classification uniquely can be spurious if we have an uneven number of observations in each class or if we have more than two classes in your data set. From this confusion matrix analysis the predicted accuracy is balanced accuracy: 0.912875 shown in confusion matrix Figure 8. In order to evaluate recognition performance of the Resnet50 algorithm the classification performance results ROC curves are shown in the Figure 9. 


\section{Training epochs includes}

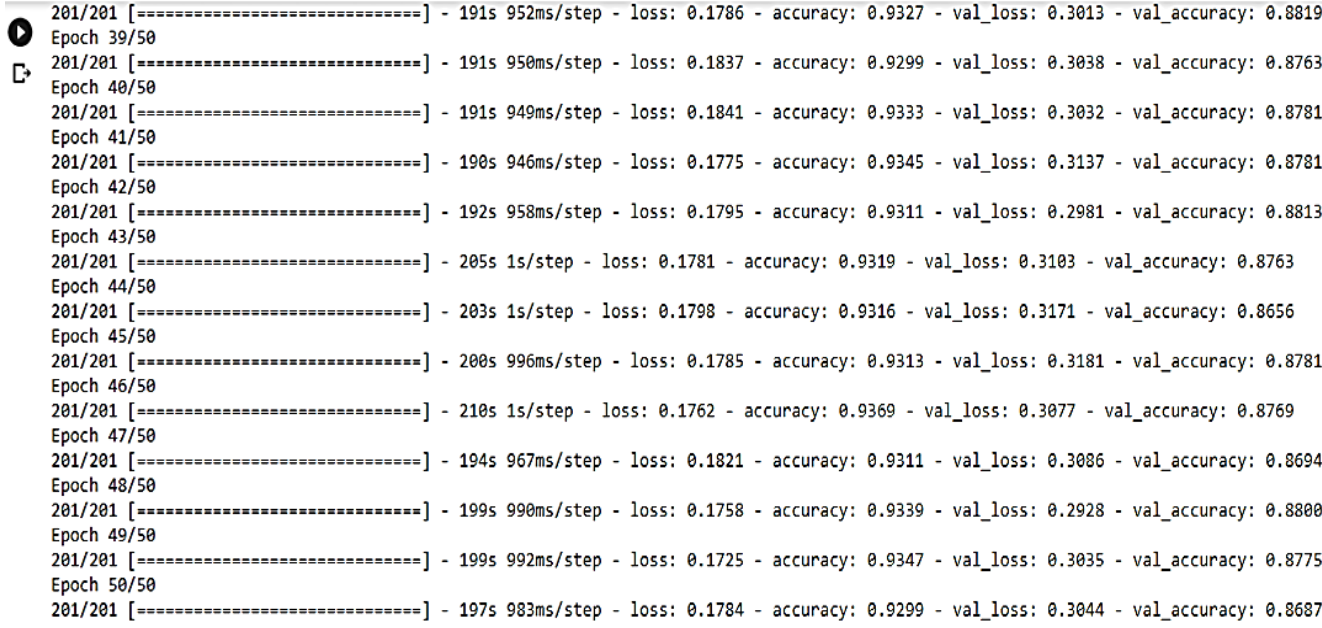

\section{Validation accuracy includes}

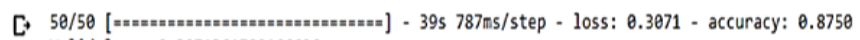

Valid loss: 0.3071361780166626

Valid acc: 0.875

Figure 7. Training epochs and validation epochs

\begin{tabular}{|c|c|c|c|c|c|c|c|}
\hline$[867$ & 120 & $\theta$ & 1 & $\theta$ & e & 9 & 3] \\
\hline [ 62 & 935 & $\theta$ & 2 & $\theta$ & $\theta$ & $\theta$ & 1] \\
\hline$\theta$ & $\theta$ & 708 & $\theta$ & 9 & 282 & $\theta$ & 1] \\
\hline 0 & $\theta$ & $\theta$ & 979 & $\theta$ & $\theta$ & 7 & 14] \\
\hline$\theta$ & $\theta$ & $\theta$ & $\theta$ & 998 & 1 & 1 & 0] \\
\hline$\theta$ & 8 & 43 & $\theta$ & 12 & 943 & 1 & 1] \\
\hline 2 & $\theta$ & $\theta$ & 40 & 13 & 1 & 890 & 54] \\
\hline$\theta$ & 1 & $\theta$ & 11 & 1 & $\theta$ & 4 & 983] \\
\hline
\end{tabular}

Figure 8. Confusion matrix

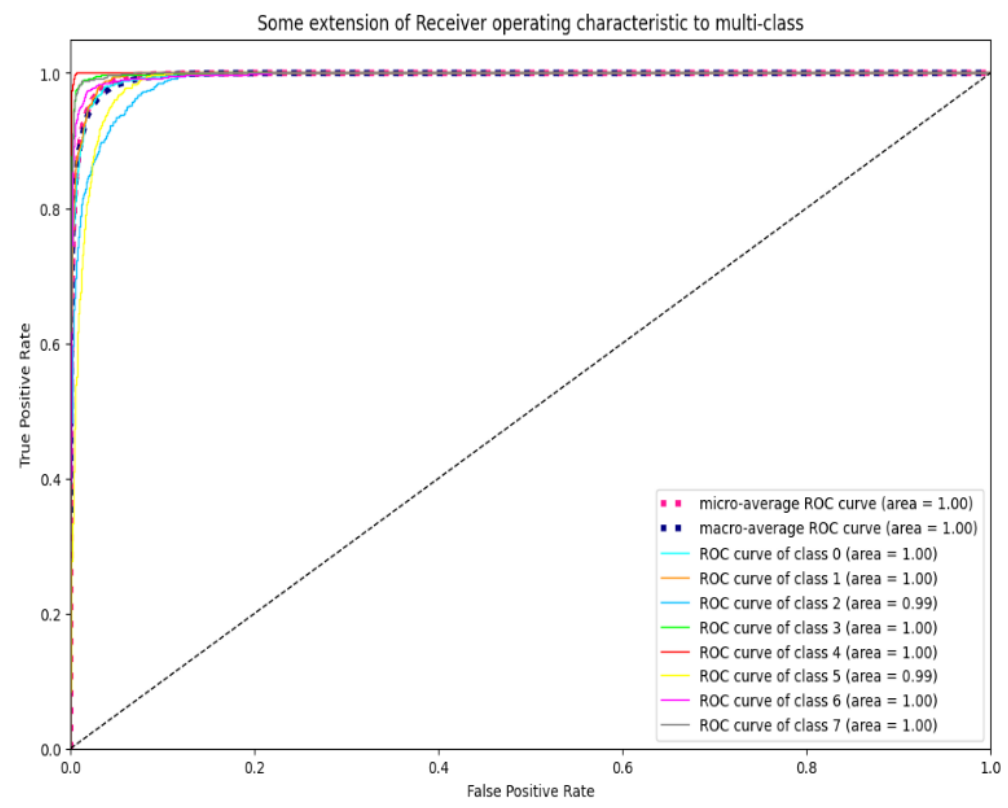

Figure 9. The ROC curve of different classification of Resnet50 algorithm 


\section{CONCLUSION}

Colorectal cancer is the largest cause of disease and death worldwide. The rigor of a successful screening method will decrease the scale of the illness caused by colorectal cancer. A variety of currently available colorectal cancer screening modalities have acceptable levels of sensitivity. As a laboratory, physicians are our goal to provide clear information and convey it to physicians. The World Health Organization informs patients about the priority of colorectal cancer screening and health care decision making. The World Health Organization delivers cost-effective testing programs in our populations and lands. This paper reviews various colorectal cancer diagnosis and prognosis techniques. This study provides a research survey on colon polyp detection victimization, many totally different approaches to screening modalities. Discuss the importance and the benefit of the setup modality like colonoscopy and the associated CAD system. When emerging from the prevalence of tumors establish in the colon and rectum and analyse their computative options, it will be guaranteed that their customs and distinct forms have been a decent sign for detecting tumors. The diagnostic issue is that the goal of lesion recognition in patients with reduced false positives and will increase positive truth that will increase accuracy and specificity. Improvements are still needed in the CAD programs studied. The classification of tumors using the CNN is discussed and mention effectively. The most important thing is that there should be an independent domain that focuses on adjusting the parameters of those methodologies. Second, the cross validation methodology may well be employed In order to achieve further statistical significance, the results and help stop overloading and optimize the performance of the CAD system oversized information.

\section{REFERENCES}

[1] Colorectal cancer screening, IARC Handbooks for Cancer Prevention, vol. 17, 2020.

[2] World Health Organization. "Global cancer observatory." International Agency for Research on Cancer. World Health Organization. https://gco.iarc.fr/ (accessed Dec. 21, 2020).

[3] M. Moshkowitz and N. Arber, "Emerging technologies in colorectal cancer screening," Surgical Oncology Clinics of North America, vol. 14, no. 4, pp. 723-746, 2005, doi: 10.1016/j.soc.2005.05.010.

[4] B. Levin, D. Brooks, R. A. Smith, and A. Stone, "Emerging technologies in screening for colorectal cancer: CT colonography, immunochemical fecal occult blood tests, and stool screening using molecular markers," CA A Cancer Journal of Clinicians, vol. 53, pp. 44-55, 2003, doi: 10.3322/canjclin.53.1.44.

[5] X. Jia, X. Xing, Y. Yuan, L. Xing and M. Q. Meng, "Wireless capsule endoscopy: a new tool for cancer screening in the colon with deep-learning-based polyp recognition," in Proceedings of the IEEE, vol. 108, no. 1, pp. 178-197, Jan. 2020, doi: 10.1109/JPROC.2019.2950506.

[6] E. Hissong and M. E. Pittman, "Colorectal carcinoma screening: Established methods and emerging technology," Critical Reviews in Clinical Laboratory Sciences, vol. 57, no. 1, pp. 22-36, 2020, doi: 10.1080/10408363.2019.1670614.

[7] I. Pacal, D. Karaboga, A. Bastruk, B. Akay, and U. Nalbantoglu, "A comprehensive review of deep learning in colon cancer," Computers in Biology and Medicine, vol. 126, 2020, Art. no. 104003, doi: 10.1016/j.compbiomed.2020.104003.

[8] H. Tang and Z. Hu, "Research on medical image classification based on machine learning," in IEEE Access, vol. 8, pp. 93145-93154, 2020, doi: 10.1109/ACCESS.2020.2993887.

[9] K. Patel et al., "A comparative study on polyp classification using convolutional neural networks," PLoS ONE, vol. 15, no. 7, 2020, doi: 0.1371/journal.pone.0236452.

[10] B. Taha, N. Werghi and J. Dias, "Automatic polyp detection in endoscopy videos: A survey," 2017 13th IASTED International Conference on Biomedical Engineering (BioMed), 2017, pp. 233-240, doi: 10.2316/P.2017.852-031.

[11] M. Makkie et al., "A distributed computing platform for fMRI big data analytics," in IEEE Transactions on Big Data, vol. 5, no. 2, pp. 109-119, 1 June 2019, doi: 10.1109/TBDATA.2018.2811508.

[12] R. Cao, Z. Tang, C. Liu and B. Veeravalli, "A scalable multicloud storage architecture for cloud-supported medical internet of things," in IEEE Internet of Things Journal, vol. 7, no. 3, pp. 1641-1654, Mar. 2020, doi: 10.1109/JIOT.2019.2946296.

[13] H. Dhayne, R. Haque, R. Kilany and Y. Taher, "In search of big medical data integration solutions - a comprehensive survey," in IEEE Access, vol. 7, pp. 91265-91290, 2019, doi: 10.1109/ACCESS.2019.2927491.

[14] L. Yu, H. Chen, Q. Dou, J. Qin and P. A. Heng, "Integrating online and offline three-dimensional deep learning for automated polyp detection in colonoscopy videos," in IEEE Journal of Biomedical and Health Informatics, vol. 21, no. 1, pp. 65-75, Jan. 2017, doi: 10.1109/JBHI.2016.2637004.

[15] Q. Li et al., "Colorectal polyp segmentation using a fully convolutional neural network," 2017 10th International Congress on Image and Signal Processing, BioMedical Engineering and Informatics (CISP-BMEI), 2017, pp. 1-5, doi: 10.1109/CISPBMEI.2017.8301980.

[16] R. Fonollà et al., "A CNN CADx system for multimodal classification of colorectal polyps combining WL, BLI, and LCI modalities," Applied Science, vol. 10, no. 15, 2020, doi: 10.3390/app10155040.

[17] Sareena, A. Mittal, and M. Kaur, "Computer-aided-diagnosis in colorectal cancer: a survey of state of the art techniques," 2016 International Conference on Inventive Computation Technologies (ICICT), 2016, pp. 1-6, doi: 10.1109/INVENTIVE.2016.7823260.

[18] W. Lie, B. Jiang, and W. Zhao, "Obstetric imaging diagnostic platform based on cloud computing technology under the background of smart medical big data and deep learning," in IEEE Access, vol. 8, pp. 78265-78278, 2020, doi: 10.1109/ACCESS.2020.2988563.

[19] T. Okamoto et al., "Feature extraction of colorectal endoscopic images for computer-aided diagnosis with CNN," 2019 2nd International Symposium on Devices, Circuits and Systems (ISDCS), 2019, pp. 1-4, doi: 10.1109/ISDCS.2019.8719104.

[20] T. Tamaki et al., "Computer-aided colorectal tumor classification in NBI endoscopy using local features," Medical Image Analysis, pp. 78-100, 2013, doi: 10.1016/j.media.2012.08.003. 
[21] T. Okamoto et al., "Implementation of computer-aided diagnosis system on customizable DSP core for colorectal endoscopic images with CNN features and SVM," TENCON 2018 - 2018 IEEE Region 10 Conference, 2018, pp. 1663-1666, doi: 10.1109/TENCON.2018.8650331.

[22] T. Yang et al., "Intelligent imaging technology in diagnosis of colorectal cancer using deep learning," in IEEE Access, vol. 7, pp. 178839-178847, 2019, doi: 10.1109/ACCESS.2019.2958124.

[23] Y. Shin, H. A. Qadir, L. Aabakken, J. Bergsland and I. Balasingham, "automatic colon polyp detection using region based deep CNN and post learning approaches," in IEEE Access, vol. 6, pp. 40950-40962, 2018, doi: 10.1109/ACCESS.2018.2856402.

[24] H. A. Qadir, J. Solhusvik, J. Bergsland, L. Aabakken, and I. Balasingham, "A framework with a fully convolutional neural network for semi-automatic colon polyp annotation," in IEEE Access, vol. 7, pp. 169537-169547, 2019, doi: 10.1109/ACCESS.2019.2954675.

[25] S. Poudel, Y. J. Kim, D. M. Vo, and S. Lee, "Colorectal disease classification using efficiently scaled dilation in convolutional neural network," in IEEE Access, vol. 8, pp. 99227-99238, 2020, doi: 10.1109/ACCESS.2020.2996770.

[26] W. Wu, "An intelligent diagnosis method of brain mri tumor segmentation using deep convolutional neural network and SVM algorithm," Computational and Mathematical Methods in Medicine, vol. 2020, 2020, Art. no. 6789306, doi: $10.1155 / 2020 / 6789306$.

\section{BIOGRAPHIES OF AUTHORS}
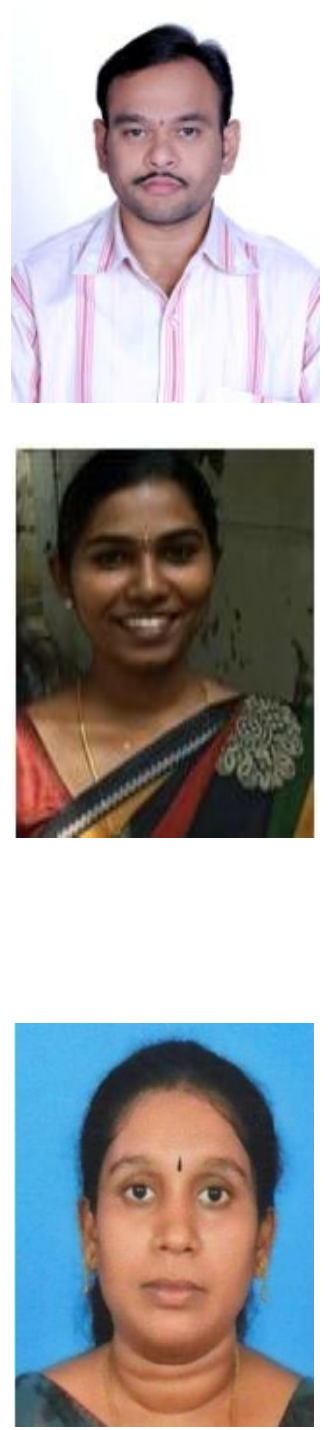

Akella S. Narasimha Raju (D) 8d SC P is a present pursuing PhD from SRM Institute of Science and Technology. He is presently doing research on the various applications of the machine learning and deep learning on health Internet of Things concept. He was 17 years of teaching experience in various organizations. He can be contacted at email: ar9488@ srmist.edu.in.

Kayalvizhi Jayavel (DD $8 \mathrm{SC}$ P is a founder IoT Alliance club, a not to profit unit to train students in the field of IoT, www.iotalliance.in. IT Coordinator for National Family Health Survey (NFHS5) for Tamilnadu-South under Ministry of Health and Family Welfare, Government of India. Published papers in reputed journals with international collaborations with countries including Japan, Oman, Rwanda, invited professor, and research supervisor at the African Centre of Excellence of Internet of Things (ACEIoT), University of Rwanda (2019 to 2020). Faculty advisor for the ACM SIGAI chapter at SRM, funded 2000 Euros by AI Journal towards the application submission "AAIW for under privileged" (2019). Mentored IoT skills for 15 Bachelor students from Amikom University, Indonesia, 2018. One of SRMSPOC in Smart India Hackathon'2018 and 2019. She is recipient of a service recognition award from ACM-W recognizes the first prize winning article published XRDS ACM magazine Dec. 2015 edition. Distinction in Cambridge certification on pedagogical study methods (2012). Visiting researcher to NEC (Japan) for a collaborative research project in the domain of WSN (2011). Recipient of SRM's best teaching performance award (2007). She can be contacted at email: kayalvij@ srmist.edu.in.

Tulasi Rajalakshmi (D) SC P $P$ is working as an assistant professor in SRM Institute of Science and Technology. She has 19 years' experience in teaching. She is a life member of ISTE, ISCA and Biomedical Engineering society. She published a number of papers in various journals. She can be contacted at email: rajalakt@ srmist.edu.in. 